\title{
Severe Acquired Coagulopathy During Fulminant Staphylococcus aureus Sepsis Most Likely Caused by S. aureus Exotoxins (SSLs)
}

\author{
$\underline{\text { Lonneke Draaijers }}^{1}$, Robert-Jan Hassing ${ }^{2}$, Menno Kooistra ${ }^{3}$, Kok van Kessel ${ }^{4}$, Marcel Hovens ${ }^{5}$ \\ ${ }^{1}$ Internal Medicine, Rijnstate Hospital Arnhem, The Netherlands \\ ${ }^{2}$ Internal Medicine, Infectious Diseases, Rijnstate Hospital Arnhem, The Netherlands \\ ${ }^{3}$ Internal Medicine, Nephrology, Rijnstate Hospital Arnhem, The Netherlands \\ ${ }^{4}$ Medical Microbiology, University Medical Center Utrecht, The Netherlands \\ ${ }^{5}$ Internal Medicine, Vascular Medicine, Rijnstate Hospital Arnhem, The Netherlands
}

\begin{abstract}
Received: 26/11/2018
Accepted: 02/12/2018

Published: $27 / 12 / 2018$
\end{abstract}

How to cite this article: Draaijers L, Hassing RJ, Kooistra M, van Kessel K, Hovens M. Severe acquired coagulopathy during fulminant staphylococcus areus sepsis most likely caused by s. aureus exotoxins (SSLs). EJCRIM 2018;5: doi:10.12890/2018_001002.

Conflicts of Interests: The Authors declare that there are no competing interests.

This article is licensed under a Commons Attribution Non-Commercial 4.0 License

\section{ABSTRACT}

Haemostatic abnormalities frequently occur during sepsis and are most often attributed to disseminated intravascular coagulation (DIC). We report the case of a patient with severe coagulopathy acquired during fulminant S. aureus sepsis. DIC was not present. This coagulopathy was most likely caused by S. aureus exotoxins forming inhibitory complexes with coagulation factor Xa.

\section{LEARNING POINTS}

- To our knowledge, this is the first report describing inhibition of coagulation by Staphylococcus aureus in vivo and so will hopefully broaden our knowledge of $S$. aureus sepsis, S. aureus exotoxins and coagulation disorders during sepsis.

- While disseminated intravascular coagulation (DIC) is the most frequent severe coagulopathy diagnosed during sepsis, inhibition of coagulation by SSL10 may be an underdiagnosed cause during S. aureus sepsis.

- As DIC and inhibition of coagulation by SSL10 should be treated differently, we emphasize the importance of considering inhibition of blood coagulation by $S$. aureus when an acquired coagulopathy is found during severe sepsis.

\section{KEYWORDS}

Staphylococcus aureus, coagulopathy, staphylococcal superantigen-like protein, coagulation factor Xa, disseminated intravascular coagulation

\section{BACKGROUND}

Staphylococcus aureus sepsis is a common cause of both hospital-acquired and community-acquired bacteraemia, with overall mortality rates of $20-40 \%{ }^{[1,2]}$. This sepsis frequently results in metastatic infections, with a negative impact on patient outcome. S. aureus infections should be treated with antibiotics, for example flucloxacillin (floxacillin).

Haemostatic abnormalities are often present during sepsis, ranging from subclinical activation of blood coagulation, to acute disseminated intravascular coagulation (DIC). DIC is characterized by massive thrombin and fibrin formation, resulting in widespread thrombosis. The administration of platelets and coagulation proteins during fulminant DIC may cause simultaneous bleeding ${ }^{[1]}$. 
A previous in vitro study showed that haemostatic abnormalities during S. aureus sepsis can also result from inhibition of blood coagulation by S. aureus itself ${ }^{[3]}$. To our knowledge, this inhibition has not yet been demonstrated in vivo.

In this case report, we present a patient with severe coagulopathy acquired during fulminant $S$. aureus sepsis, most likely caused by inhibition of coagulation factor $\mathrm{X}$ by $\mathrm{S}$. aureus exoproteins.

\section{CASE PRESENTATION}

A 60-year-old man with a history of diabetes mellitus type 2, was admitted to our hospital with symptoms of coughing and fever. An x-ray of the thorax showed an infiltrate of the left lung. Treatment with amoxicillin and ciprofloxacin was initiated for severe pneumonia. Two days after admission, blood cultures showed S. aureus. Medication was switched to flucloxacillin. No metastatic infections were found on PET-CT imaging combined with transoesophageal echocardiography. The patient developed atrial fibrillation, for which rivaroxaban (20 mg once daily) was initiated. However, 6 days after initiation, the rivaroxaban was stopped because of a decline in renal function.

Five days after the cessation of rivaroxaban and 15 days after the first blood cultures showing S. aureus, blood coagulation tests were performed, preceding a kidney biopsy. These coagulation tests revealed a partial thromboplastin time (PT) of over 90 sec and an activated partial thromboplastin time (aPTT) of $83 \mathrm{sec}$. Apart from an episode of haematuria, our patient did not have any bleeding complications. PT and aPTT values from the day of hospital admission (day 0) to 3 months afterwards, as well as other laboratory values, are shown in Table 1. Further investigation of the coagulation revealed no signs of DIC. Mixing studies showed complete normalization of clotting times, thus excluding the presence of a clotting factor inhibitor. Coagulation factor activity tests revealed normal activity of clotting factors V, VII and VIII, and a deficiency of clotting factor X (Table 1). Lastly, the serum drug concentration of rivaroxaban on day 16 was $2 \mu \mathrm{g} / \mathrm{l}$, which is far below the therapeutic range ${ }^{[4]}$. As shown in Table 1, both the PT and aPTT normalized spontaneously 3 weeks after hospital admission.

\begin{tabular}{|c|c|c|c|c|c|c|c|c|}
\hline \multirow[t]{2}{*}{ Laboratory values } & \multicolumn{8}{|c|}{ Number of days after admission to hospital } \\
\hline & 0 & $8^{*}$ & 15 & 16 & 18 & 22 & $24 \dagger \S$ & $94 \dagger$ \\
\hline Haemoglobin, $\mathrm{mmol} / \mathrm{l}$ & 9.4 & 8.6 & 7.8 & 7.5 & 7.7 & 7.0 & 6.4 & 7.0 \\
\hline Platelets, $\times 10^{9} / 1$ & 165 & 344 & 328 & 300 & 320 & 223 & 186 & 219 \\
\hline aPTT, sec & 39 & 41 & 83 & 87 & 63 & 43 & 53 & 42 \\
\hline PT, sec & 18 & 20 & $>90$ & $>90$ & 34 & 18 & 23 & 25 \\
\hline Creatinine, $\mu \mathrm{mol} / / \mathrm{I}$ & 90 & 346 & 692 & 702 & 674 & 450 & 294 & 111 \\
\hline$e G F R, \mathrm{ml} / \mathrm{min} / 1.73 \mathrm{~m}^{2}$ & 79 & 16 & 7 & 7 & 7 & 11 & 19 & 58 \\
\hline Fibrinogen, g/l & & & & 5 & & & & \\
\hline Factor V activity, \% & & & & 129 & & & & \\
\hline Factor VII activity, \% & & & & $>200$ & & & & \\
\hline Factor VIII activity, \% & & & & $>200$ & & & & \\
\hline Factor X activity, \% & & & & 12 & & & & \\
\hline
\end{tabular}

Table 1. Laboratory values after admission to hospital

${ }^{*}$ During treatment with rivaroxaban (treatment with rivaroxaban $20 \mathrm{mg}$ once daily was given between day 4 and day 10 after admission to hospital).

†During treatment with acenocoumarol.

§ay of hospital discharge.

Normal values: haemoglobin 8.4-10.8 mmol/l; platelets 150-400 ×10\%/l; aPTT 24-34 sec; PT 12-15 sec; creatinine 60-110 $\mu \mathrm{mol} / \mathrm{l} ;$ eGFR >90 ml/min/1.73m² (calculated with the CKD-EPI equation); fibrinogen 2-4 g/l; factor V activity 70-150\%; factor VII activity 60-140\%; factor VIII activity 50-200\%; factor X activity 60-140\%. 


\section{DISCUSSION}

We have described the case of a patient with severe coagulopathy acquired during fulminant S. aureus sepsis. A solitary deficiency of factor $X$ was found and other causes of combined PT and aPTT prolongation were excluded. The coagulopathy was most likely caused by inhibition of coagulation factor $\mathrm{X}$ by the $S$. aureus exotoxins staphylococcal superantigen-like proteins (SSLs). The bleeding disorder disappeared simultaneously with resolution of the sepsis.

Fig. 1 shows a simplified version of the coagulation cascade. As a solitary deficiency of factor $\mathrm{X}$ was found, the acquired coagulopathy was less likely caused by liver disease, vitamin K deficiency, DIC, warfarin intoxication or rodenticide poisoning. The rivaroxaban blood drug concentration was subtherapeutic, excluding anticoagulant overdosage. A factor $\mathrm{X}$ inhibitor was excluded and there were no signs of rapid-onset amyloid light-chain amyloidosis, or multiple myeloma. A previous literature study showed S. aureus is able to inhibit host blood coagulation by inhibiting clotting factor $X^{[3]}$. As all other possible causes of an acquired factor $X$ deficiency were excluded, we concluded that inhibition of clotting factor $X$ by $S$. aureus was the most likely cause of the acquired coagulopathy in our patient.

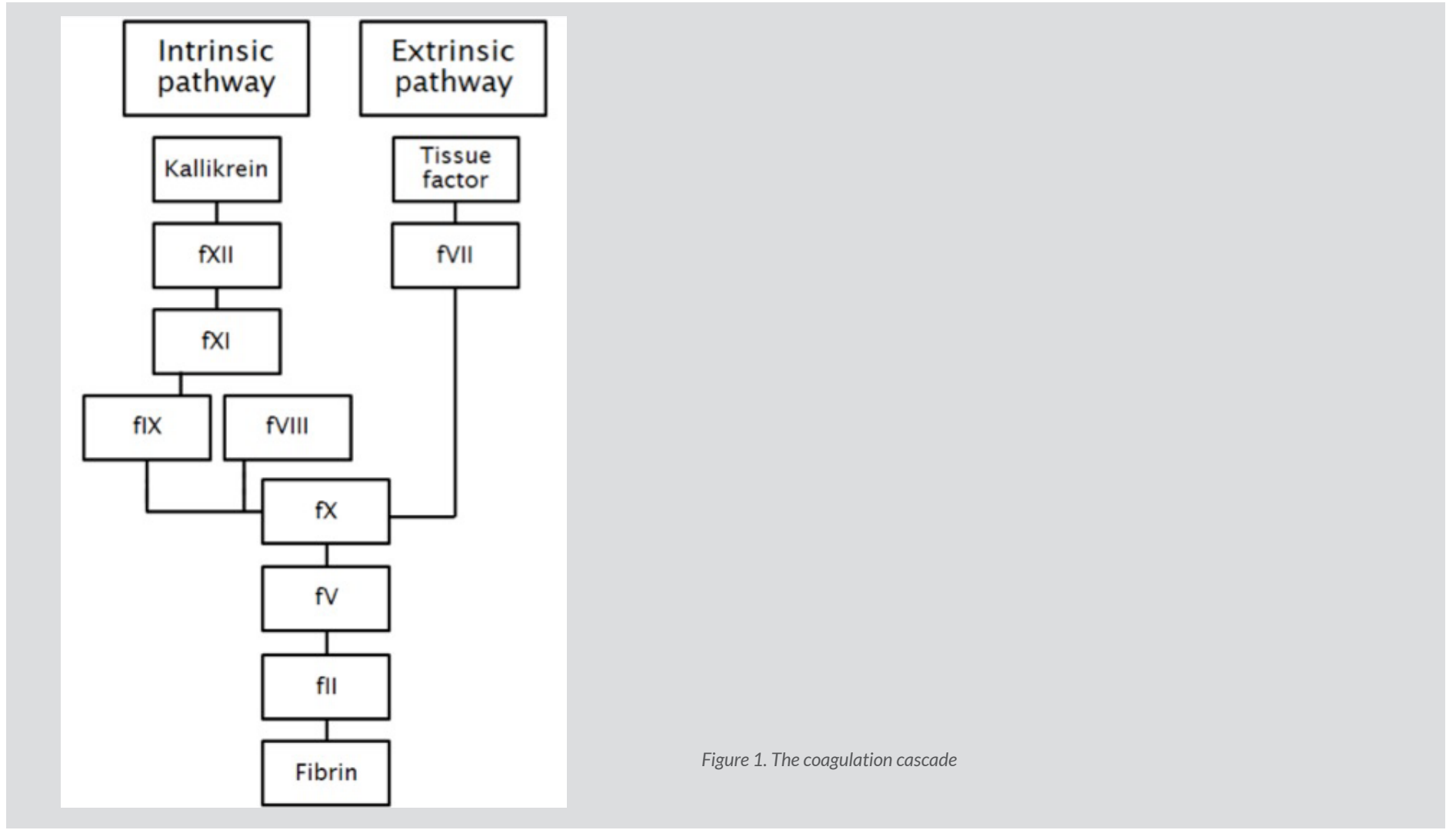

Superantigens (SAgs) and SSLs are exoproteins produced by S. aureus. SAgs exhibit superantigenic activity, which means they act by causing activation of T cells. They are important in a variety of diseases, including toxic shock syndrome (TSS), Kawasaki disease, staphylococcal pneumoniae and staphylococcal purpura fulminans ${ }^{[2]}$. SSLs do not exhibit superantigenic activity. Earlier studies demonstrated that SSLs are able to disturb host immune functions and inhibit blood coagulation in a T-cell-independent way ${ }^{[3]}$.

Several SSLs disturb host immune function. For example, SSL3 binds to Toll-like receptor 2 and inhibits cytokine production by macrophage ${ }^{[5,6]}$, while SSL10 inhibits the classical complement activation pathway, the migration of T cells, the interaction between complement C1q and $\operatorname{lgG}$, and the Fc-receptor-mediated phagocytosis of neutrophils ${ }^{[7-9]}$.

Previous in vitro studies showed that SSL10 is also able to inhibit blood coagulation ${ }^{[3]}$. This exoprotein binds specifically to coagulation factor Xa and prothrombin, two major factors involved in the blood coagulation cascade (Fig. 1). Due to this binding, the generation of thrombin is reduced, blood coagulation is inhibited and clotting times are prolonged in a dose-dependent way.

Since extreme prolongation of clotting times was found in our patient, we believe he was infected with a strain of $S$. aureus which produced more SSL10 than average. However, we could not find a circulating inhibitor of factor $X$, since mixing studies revealed complete normalisation of clotting times. 
We speculate that all of the SSL10 was already bound to factor $\mathrm{X}$ when coagulation tests were carried out. In an attempt to detect the presence of SSL10 in the in vitro culture supernatant of this strain, we performed SDS-PAGE and Western blot analysis with an SSL10specific antiserum. These tests did not detect the presence of SSL10 in the bacterial culture supernatant under these conditions. It is known that the detection of many SSLs in the culture supernatants of various strains and under different conditions in vitro is difficult, most likely caused by low levels of secretion in vitro ${ }^{[10]}$. This may be the reason why we were unable to detect the presence of SSL10 in the bacterial supernatant, and does not exclude the presence of significant in vivo levels.

Inhibition of coagulation factor X by S. aureus SSL10 is the most likely cause of the acquired coagulopathy in our patient. To our knowledge, no earlier reports have described this coagulopathy in vivo. While DIC is the most frequently diagnosed severe coagulopathy during sepsis, inhibition of coagulation by SSL10 may be an underdiagnosed cause during S. aureus sepsis.

A solitary factor $X$ deficiency with bleeding complications is best treated with coagulation factor concentrate, while treatment of DIC should primarily focus on addressing the underlying cause. Therefore, we emphasize the importance of considering inhibition of blood coagulation by S. aureus when an acquired coagulopathy is found during severe sepsis.

\section{CONCLUSION}

We have described a patient with severe coagulopathy acquired during fulminant S. aureus sepsis, most likely caused by S. aureus exotoxins forming inhibitory complexes with coagulation factor $\mathrm{X}$. To our knowledge, this is the first demonstration of this inhibition in humans. However, this may be a more common and underdiagnosed cause of acquired coagulopathy during S. aureus sepsis. As treatment of a solitary factor X deficiency is different from the treatment of DIC, we emphasize the importance of considering inhibition of blood coagulation by $S$. aureus when acquired coagulopathy is found during severe sepsis.

\section{REFERENCES}

1. Semeraro N, Ammollo CT, Semeraro F, Colucci M. Sepsis-associated disseminated intravascular coagulation and thromboembolic disease. Mediterr J Hematol Infect Dis 2010;2:e2010024.

2. Shurland S, Zhan M, Bradham DD, Roghmann MC. Comparison of mortality risk associated with bacteremia due to methicillin-resistant and methicillin-susceptible staphylococcus aureus. Infection control and hospital epidemiology. Infect Control Hosp Epidemiol 2007;28:273-279.

3. Itoh S, Yokoyama R, Kamoshida G, Fujiwara T, Okada H, Takii T, et al. Staphylococcal superantigen-like protein 10 (SSL10) inhibits blood coagulation by binding to prothrombin and factor Xa via their gamma-carboxyglutamic acid (Gla) domain. J Biol Chem 2013;288:21569-21580.

4. Samama MM, Contant G, Spiro TE, Perzborn E, Le Flem L, Guinet C, et al. Laboratory assessment of rivaroxaban: a review. Thromb J 2013;11:11.

5. Yokoyama R, Itoh S, Kamoshida G, Takii T, Fujii S, Tsuji T, et al. Staphylococcal superantigen-like protein 3 binds to the Toll-like receptor 2 extracellular domain and inhibits cytokine production induced by staphylococcus aureus, cell wall component, or lipopeptides in murine macrophages. Infect Immun 2012;80:2816-2825.

6. Bardoel BW, Vos R, Bouman T, Aerts PC, Bestebroer J, Huizinga EG, et al. Evasion of Toll-like receptor 2 activation by staphylococcal superantigen-like protein 3. J Mol Med (Berl) 2012;90:1109-1120.

7. Walenkamp AM, Boer IG, Bestebroer J, Rozeveld D, Timmer-Bosscha H, Hemrika W, et al. Staphylococcal superantigen-like 10 inhibits CXCL12-induced human tumor cell migration. Neoplasia 2009;11:333-344.

8. Itoh S, Hamada E, Kamoshida G, Yokoyama R, Takii T, Onozaki K, et al. Staphylococcal superantigen-like protein 10 (SSL10) binds to human immunoglobulin G (IgG) and inhibits complement activation via the classical pathway. Mol Immunol 2010;47:932-938.

9. Patel D, Wines BD, Langley RJ, Fraser JD. Specificity of staphylococcal superantigen-like protein 10 toward the human IgG1 Fc domain. J Immunol 2010;184:6283-6292.

10. den Reijer PM, Lemmens-den Toom N, Kant S, Snijders SV, Boelens H, Tavakol M, et al. Characterization of the humoral immune response during staphylococcus aureus bacteremia and global gene expression by staphylococcus aureus in human blood. PLoS One 2013;8:e53391. 